\title{
Quantum Current Operators-II Difference Equations of Quantum Current Operators and Quantum Parafermion Construction
}

\author{
By \\ Jintai Ding* and Boris FeIGIN**
}

\begin{abstract}
For the current realization of the affine quantum groups, a simple comultiplication for the quantum current operators was given by Drinfeld. With this comultiplication, we prove that, for the integrable modules of $U_{q}(\hat{\mathfrak{g} l}(2))$ of level $k+1, x^{ \pm}(z) x^{ \pm}\left(z q^{ \pm 2}\right) \cdots x^{ \pm}\left(z q^{ \pm 2 k}\right)$ are vertex operators satisfying certain q-difference equations, and we derive the quantum parafermions of $U_{q}(\hat{\mathfrak{s} l}(2))$.
\end{abstract}

\section{§1. Introduction}

Lie algebra $\hat{\mathfrak{s} l}(2)$ has three current operators $e(z), h(z)$ and $f(z)$. For any integrable highest weight module of $\hat{\Xi} l(2)$ of level $k$, the current operators $e(z)$ and $f(z)$ satisfy the following differential equation:

$$
\left(e^{k}(z)\right)^{\prime}-: z^{-1} h(z) e^{k}(z):=0=\left(f^{k}(z)\right)^{\prime}+: z^{-1} h(z) f(z):
$$

which implies that $e^{k}(z)$ and $f^{k}(z)$ are vertex operators [LP].

For the case of quantum affine algebras, Drinfeld presented a formulation of affine quantum groups with generators in the form of current operators [Dr2], which, for the case of $U_{q}(\hat{\mathfrak{b} l} l(2))$, give us the quantized current operators corresponding to $e(z), h(z)$ and $f(z)$ of $\hat{\xi} l(2)$. We would like to find out if it is possible to derive a similar equation, which will degenerate into the equation above. To solve this problem, we need to use the Drinfeld comultiplication for the current formulation of $U_{q}(\hat{\mathfrak{S} l}(2))[\mathrm{DF}][\mathrm{DI}]$, which resolve the difficulty caused by the non-commutativity of those quantum current operators. This comultiplication is very simple as opposed to the comultiplication formula

Communicated by M. Kashiwara, September 10, 1996.

1991 Math. Subject Classification(s) : 17B37

*RIMS, Kyoto University, Kyoto, 606-01, Japan.

**RIMS, Kyoto University; Landau Institute, Moscow. 
induced from the conventional comultiplication, which can not be written in a closed form with those current operators. With this comultiplication, we are able to study the zeros and poles of quantum current operators for integrable modules to derive a quantum integrable condition for $U_{q}(\hat{\mathfrak{g} l}(2))[\mathrm{DM}]$.

In this paper, we will use the same method as in [DM]. We will start with the case of the module $F$ for the fundamental representations at the level 1 for the case of $U_{q}(\hat{\mathfrak{g l} l}(2))$, which is the representation constructed by Frenkel and Jing by using vertex operators. $U_{q}(\hat{\mathfrak{g} l} l(2))$ as in the Drinfeld realization (see Definition 2.1) has four current generators $x^{+}(z), \varphi(z), \psi(z)$ and $x^{-}(z)$, where $x^{+}(z)$ and $x^{-}(w)$ are quantized current operators of $U_{q}(\hat{\mathfrak{g} l} l(2))$ corresponding to $e(z)$ and $f(z)$ of $\hat{s} l(2)$ respectively and $\varphi(z), \psi(z)$ are quantized current operator corresponding to the negative half and the positive half of $h(z)$ of $\hat{\mathfrak{s} l}(2)$ respectively. Using Drinfeld comultiplication, we show that, on any level $m+1$ integrable module of $U_{q}(\hat{\mathfrak{g} l}(2))$,

$$
\begin{gathered}
\left(x^{+}\left(z q^{2}\right)\right)\left(x^{+}\left(z q^{4}\right)\right) \cdots\left(x^{+}\left(z q^{(2 m+2)}\right)\right)= \\
\varphi^{-1}\left(z q^{m / 2+1 / 2}\right)\left(x^{+}(z)\right)\left(x^{+}\left(z q^{2}\right)\right) \cdots\left(x^{+}\left(z q^{(2 m)}\right)\right) \phi\left(z q^{(m+1) 3 / 2}\right), \\
\left(x^{-}\left(z q^{2 m+2}\right)\right)\left(x^{-}\left(z q^{2 m}\right)\right) \cdots\left(x^{-}\left(z q^{2}\right)\right)= \\
\varphi\left(z q^{-(m+1) 3 / 2}\right)\left(x^{-}\left(z q^{2 m}\right)\right)\left(x^{-}\left(z q^{2 m-2}\right)\right) \cdots\left(x^{-}(z)\right) \phi^{-1}\left(z q^{-m / 2+1 / 2}\right),
\end{gathered}
$$

and, $\left(x^{+}(z)\right) \cdots\left(x^{+}\left(z q^{(2 m)}\right)\right)$ and $\left(x^{-}\left(z q^{(2 m)}\right)\right)\left(x^{-}\left(z q^{2 m-2}\right)\right) \cdots\left(x^{-}(z)\right)$ are vertex operators. In the last section, we apply this method to derive the parafermions $\phi_{i}(z), i=0, \ldots, m-1$, on the module $\otimes^{m+1} \mathrm{~F}$ for $U_{q}(\hat{\mathfrak{g} l} l(2))$, such that

$$
\begin{gathered}
\left(x^{+}(z)\right)\left(x^{+}\left(z q^{2}\right) \cdots\left(x^{+}\left(z q^{2 k}\right)\right)=V_{k}^{+}(z) \phi^{m, k}(z),\right. \\
\left(x^{-}\left(z q^{2 k}\right)\right)\left(x^{+}\left(z q^{2 k-2}\right)\right) \cdots\left(x^{+}(z)\right)=V_{k}^{-}(z) \phi_{m, m-k-1}(z),
\end{gathered}
$$

where $\phi_{m, i}(z)$ commute with $\varphi(z)$ and $\phi(z)$, and $V_{k}^{+}(z)$ and $V_{k}^{-}(z)$ are vertex operators.

\section{§2. Quantum Difference Equation and Vertex Operator} [Dr2].

We will first present the current realization of $U_{q}\left(\hat{\mathfrak{G l}}_{2}\right)$ given by Drinfeld

Definition 2.1. The algebra $U_{q}\left(\hat{\mathfrak{S}}_{2}\right)$ is an associative algebra with unit 1 and the generators : $\varphi(m), \psi(-m), x^{ \pm}(l)$, for $i=1, \ldots, n-1, l \in \mathbf{Z}$ and $m \in \mathbf{Z}_{\leq 0}$ and a central element $c$. Let $z$ be a formal variable and $x^{ \pm}(z)=\sum_{l \in \mathbf{Z}} x^{ \pm}(l) z^{-l}$, $\varphi(z)=\sum_{m \in \mathbf{Z}_{\leq 0}} \varphi(m) z^{-m}$ and $\phi(z)=\sum_{m \in \mathbf{Z}_{\geq 0}} \psi(m) z^{-m}$. In terms of the formal variables, the defining relations are

$$
\varphi(0) \phi(0)=\phi(0) \varphi(0)=1
$$




$$
\begin{aligned}
& \varphi(z) \varphi(w)=\varphi(w) \varphi(z), \\
& \psi(z) \psi(w)=\psi(w) \psi(z), \\
& \varphi(z) \psi(w) \varphi(z)^{-1} \psi(w)^{-1}=\frac{g\left(z / w q^{-c}\right)}{g\left(z / w q^{c}\right)}, \\
& \varphi(z) x^{ \pm}(w) \varphi(z)^{-1}=g\left(z / w q^{\mp \frac{1}{2} c}\right)^{ \pm 1} x^{ \pm}(w), \\
& \psi(z) x^{ \pm}(w) \psi(z)^{-1}=g\left(w / z q^{\mp \frac{1}{2} c}\right)^{\mp 1} x^{ \pm}(w), \\
& {\left[x^{+}(z), x^{-}(w)\right]=\frac{1}{q-q^{-1}}\left\{\delta\left(z / w q^{-c}\right) \psi\left(w q^{\frac{1}{2} c}\right)-\delta\left(z / w q^{c}\right) \varphi\left(z q^{\frac{1}{2} c}\right)\right\}} \\
& \left(z-q^{ \pm a} w\right) x^{ \pm}(z) x^{ \pm}(w)=\left(q^{ \pm a} z-w\right) x^{ \pm}(w) x^{ \pm}(z),
\end{aligned}
$$

where

$$
\delta(z)=\sum_{k \in \mathbf{Z}} z^{k}, g(z)=\frac{q^{a} z-1}{z-q^{a}}(\text { expanded around } z=0), a=2
$$

For this current realization, Drinfeld also gave the Hopf algebra structure.

Theorem 2.1. The algebra $U_{q}\left(\hat{\mathfrak{G I}}_{2}\right)$ has a Hopf algebra structure, which are given by the following formulae.

\section{Coproduct $\triangle$}

(0) $\Delta\left(q^{c}\right)=q^{c} \otimes q^{c}$,

(1) $\Delta\left(x^{+}(z)\right)=x^{+}(z) \otimes 1+\varphi\left(z q^{\frac{c_{1}}{2}}\right) \otimes x^{+}\left(z q^{c_{1}}\right)$,

(2) $\Delta\left(x^{-}(z)\right)=1 \otimes x^{-}(z)+x^{-}\left(z q^{c_{2}}\right) \otimes \psi\left(z q^{\frac{c_{2}}{2}}\right)$,

(3) $\Delta(\varphi(z))=\varphi\left(z q^{-\frac{c_{2}}{2}}\right) \otimes \varphi\left(z q^{\frac{c_{1}}{2}}\right)$,

(4) $\Delta(\psi(z))=\psi\left(z q^{\frac{c_{2}}{2}}\right) \otimes \psi\left(z q^{-\frac{c_{1}}{2}}\right)$,

where $c_{1}$ is the action of the central element $c$ on the first component and $c_{2}$ is the action of the central element $c$ on the second component.

Counit $\varepsilon$

$$
\begin{aligned}
& \varepsilon\left(q^{c}\right)=1 \quad \varepsilon(\varphi(z))=\varepsilon(\phi(z))=1, \\
& \varepsilon\left(x^{ \pm}(z)\right)=0 \text {. }
\end{aligned}
$$

Antipode a

$$
\begin{aligned}
& \text { (0) } a\left(q^{c}\right)=q^{-c}, \\
& \text { (1) } a\left(x^{+}(z)\right)=-\varphi\left(z q^{-\frac{c}{2}}\right)^{-1} x^{+}\left(z q^{-c}\right), \\
& \text { (2) } a\left(x^{-}(z)\right)=-x^{-}\left(z q^{-c}\right) \psi\left(z q^{-\frac{c}{2}}\right)^{-1}, \\
& \text { (3) } a(\varphi(z))=\varphi(z)^{-1}, \\
& \text { (4) } a(\phi(z))=\psi(z)^{-1} .
\end{aligned}
$$

This comultiplication structure requires certain completion on the tensor space. For certain representations, such as the $n$-dimensional representatios of 
$U_{q}\left(\hat{\mathfrak{G l}}_{n}\right)$ at a special value, this comultiplication may not be well-defined. Nevertheless, for any two highest weight representations, this comultiplication is well-defined, because the action of the operator as a coefficient of $z^{m}$ of the currents operators on any element of such a module are zero if $m$ is small enough.

We will present the Frenkel-Jing construction of level 1 representation of $U_{q}\left(\hat{\mathfrak{S}}_{2}\right)$ on the Fock space.

Consider an algebra generated by $\left\{a_{k} \mid k \in \mathbb{Z} \backslash\{0\}\right\}$ satisfying :

$$
\left[a_{k}, a_{l}\right]=\delta_{k+l, 0} \frac{[2 k][k]}{k}
$$

where $[k]=\frac{q^{k}-q^{-k}}{q-q^{-1}}$. We call it the Heisenberg algebra.

Let $\bar{Q}=\mathbf{Z} \alpha$ be the root lattice of $\xi l(2)$. Let us define a group algebra $\mathbf{C}(q)[\overline{\mathcal{P}}]$, where $\overline{\mathcal{P}}$ is the weight lattice of $\mathfrak{S l}_{2}$. Let $\Lambda_{1}$ be the fundamental weight of $\mathfrak{s l}(2)$ and $2 \Lambda_{1}=a$. Let $\Lambda_{0}=0$.

Set

$$
\mathscr{F}_{i}:=\mathbf{C}(q)\left[a_{-k}\left(k \in \mathbf{Z}_{>0}\right)\right] \otimes \mathbf{C}(q)[\bar{Q}] e^{\bar{\Lambda}_{i}} .
$$

This gives the Fock space.

The action of operators $a_{k}, \partial_{\alpha}, e^{\alpha}(1 \leq j \leq N)$ is given by

$$
\begin{aligned}
& a_{k} \cdot f \otimes e^{\beta}= \begin{cases}a_{k} f \otimes e^{\beta} & k<0 ; \\
{\left[a_{k}, f\right] \otimes e^{\beta}} & k>0,\end{cases} \\
& \partial_{\alpha} \cdot f \otimes e^{\beta}=(\alpha, \beta) f \otimes e^{\beta} \\
& e^{\alpha} \cdot f \otimes e^{\beta}=f \otimes e^{\alpha} e^{\beta} .
\end{aligned}
$$

Lemma 2.2. The following action on $\mathscr{F}_{i}$ of $U_{q}(\hat{\mathfrak{b} l}(2))$ gives a level 1 highest weight representation with the $i$-th fundamental weight.

$$
\begin{aligned}
\circ x^{ \pm}(z) & \mapsto \exp \left[ \pm \sum_{k>0} \frac{a_{-k}}{[k]} q^{\mp \frac{1}{2} k} z^{k}\right] \exp \left[\mp \sum_{k>0} \frac{a_{k}}{[k]} q^{\mp \frac{1}{2} k} z^{-k}\right] e^{ \pm \alpha} z^{ \pm \alpha \alpha+1} \\
\circ \varphi(z) & \mapsto \exp \left[-\left(q-q^{-1}\right) \sum_{k>0} a_{-k} z^{-k}\right] q^{-\partial \alpha} \\
\circ \phi(z) & \mapsto \exp \left[\left(q-q^{-1}\right) \sum_{k>0} a_{k} z^{-k}\right] q^{\partial \alpha}
\end{aligned}
$$

This implies that on $\mathscr{F}_{i}$ for the case of $U_{q}\left(\hat{\mathfrak{L}}_{2}\right)$

$$
\begin{aligned}
& x^{+}(z) x^{+}(w)=z^{2}\left(1-\frac{w}{z}\right)\left(1-\frac{w}{z q^{2}}\right): x^{+}(z) x^{+}(w): \\
& x^{-}(z) x^{-}(w)=z^{2}\left(1-\frac{w}{z}\right)\left(1-\frac{w q^{2}}{2}\right): x^{-}(z) x^{-}(w):
\end{aligned}
$$




$$
\begin{gathered}
x^{+}(z) \varphi(w)=q^{-2} \frac{\left(\frac{w q^{-1 / 2}}{z}-q^{2}\right)}{\left(\frac{w q^{5 / 2}}{z}-1\right)}: \varphi(w) x^{+}(z):=\frac{\left(\frac{w q^{-1 / 2}}{z}-q^{2}\right)}{\left(\frac{w q^{5 / 2}}{z}-1\right)} \varphi(w) x^{+}(z) \\
\psi(w) x^{-}(z)=\frac{\left(\frac{z q^{1 / 2}}{w}-q^{2}\right)}{\left(\frac{z q^{1 / 2}}{w}-q^{2}\right)}: \phi(w) x^{-}(z):=\frac{\left(\frac{z q^{1 / 2}}{w}-q^{2}\right)}{\left(\frac{z q^{5 / 2}}{w}-1\right)} x^{-}(z) \psi(w)
\end{gathered}
$$

Lemma 2.3. Set $F=\sum \oplus \mathscr{F}_{i}$. Any level $m$ integrable module is a submodule of $\otimes^{m} F$.

For the case of $\hat{\mathfrak{s l}}(2)$, we have that the correlation functions of $e(z) e(w)$ and $f(z) f(w)$ have no poles, which are always polynomials of $z, z^{-1}, w, w^{-1}$. By the correlation functions of an operator, we mean all the matrix coefficients of the operator. However, for the quantum case, we have [DM]

Theorem 2.4. For any level $m>1$ integrable module of $U_{q}\left(\hat{\mathfrak{G l}}_{2}\right)$, the correlation functions of $x^{+}(z) x^{+}(w)$ has at most poles at $z q^{-2}=w$. For any level $m>1$ integrable module of $U_{q}\left(\hat{\mathfrak{S}}_{2}\right)$, the correlation functions of $x^{-}(z) x^{-}(w)$ has at most poles at $z q^{2}=w$.

Theorem 2.5. For any level $m$ integrable module $U_{q}\left(\hat{\mathfrak{S}}_{2}\right)$, the correlation functions of $x^{+}\left(z_{m+1}\right) x^{+}\left(z_{m}\right) \ldots x^{+}\left(z_{2}\right) x^{+}\left(z_{1}\right)$ is zero at $z_{i} / z_{i+1}=q^{2}$. For any level $m$ integrable module of $U_{q}\left(\hat{\mathfrak{S} \mathfrak{L}_{2}}\right)$, the correlation functions of $x^{-}\left(z_{m+1}\right) x^{-}\left(z_{m}\right)$ $\ldots x^{-}\left(z_{2}\right) x^{-}\left(z_{1}\right)$ is zero, if $z_{i+1} / z_{i}=q^{2}$.

Lemma 2.6. On $F$, we have

$$
\begin{gathered}
x^{ \pm}\left(q^{2} z\right)=q^{ \pm \partial \alpha} \exp \left[ \pm \sum_{k>0} a_{-k}\left(q-q^{-1}\right) q^{k} q^{\mp \frac{1}{2} k} z^{k}\right] x^{ \pm}(z) \times \\
q^{ \pm \partial \alpha} \exp \left[\mp \sum_{k>0}\left(q-q^{-1}\right)\left(-q^{-k}\right) a_{k} q^{\mp \frac{1}{2} k} z^{-k}\right]= \\
\varphi^{\mp 1}\left(z q^{\mp \frac{1}{2}+1}\right) x^{ \pm}(z) \psi^{ \pm 1}\left(z q^{ \pm \frac{1}{2}+1}\right) .
\end{gathered}
$$

Proof.

$$
\begin{aligned}
& x^{ \pm}\left(q^{2} z\right)=\exp \left[ \pm \sum_{k>0} \frac{a_{-k}}{[k]} q^{\mp \frac{1}{2} k} q^{2 k} z^{k}\right] \exp \left[\mp \sum_{k>0} \frac{a_{k}}{[k]} q^{\mp \frac{1}{2} k} q^{-2 k} z^{-k}\right] e^{ \pm \alpha} z^{ \pm \partial \alpha+1} q^{2 \pm \partial \alpha} q^{2} \\
& =\exp \left[ \pm \sum_{k>0} \frac{a_{-k}}{[k]} q^{\mp \frac{1}{2} k}\left(q^{2 k}-1\right) z^{k}\right] x^{ \pm}(z) \exp \left[\mp \sum_{k>0} \frac{a_{k}}{[k]} q^{\mp \frac{1}{2} k}\left(q^{-2 k}-1\right) z^{-k}\right] q^{2} q^{2 \pm \partial \alpha}
\end{aligned}
$$




$$
\begin{aligned}
= & \exp \left[ \pm \sum_{k>0} a_{-k}\left(q-q^{-1}\right) q^{n} q^{\mp \frac{1}{2} k} z^{k}\right] x^{ \pm}(z) \\
& \exp \left[\mp \sum_{k>0}\left(q-q^{-1}\right)\left(-q^{-n}\right) a_{k} q^{\mp \frac{1}{2} k} z^{-k}\right] q^{2} q^{2 \pm \partial \alpha} \\
= & q^{ \pm \partial \alpha} \exp \left[ \pm \sum_{k>0} a_{-k}\left(q-q^{-1}\right) q^{k} q^{\mp \frac{1}{2} k} z^{k}\right] x^{ \pm}(z) q^{ \pm \partial_{\alpha}} \\
& \exp \left[\mp \sum_{k>0}\left(q-q^{-1}\right)\left(-q^{-k}\right) a_{k} q^{\mp \frac{1}{2} k} z^{-k}\right] .
\end{aligned}
$$

Lemma 2.7. On $\otimes^{m+1} F$, let, $\Delta^{m}\left(x^{+}(z)\right)=(1 \otimes 1 \otimes \ldots \otimes \Delta) \ldots(1 \otimes \Delta)(\Delta)$ $\left(\left(x^{+}\right)(z)\right)$, then

$$
\begin{gathered}
\Delta^{m}\left(x^{+}(z)\right) \Delta^{m}\left(x^{+}\left(z q^{2}\right)\right) \cdots \Delta^{m}\left(x^{+}\left(z q^{(2 m)}\right)\right)= \\
\varphi\left(z q^{1 / 2}\right) \varphi\left(z q^{1 / 2+2}\right) \cdots \varphi\left(z q^{1 / 2+2 m-2}\right) x^{+}\left(z q^{2 m}\right) \otimes \varphi\left(z q^{3 / 2}\right) \varphi\left(z q^{3 / 2+2}\right) \\
\cdots \varphi\left(z q^{3 / 2+2 m-4}\right) x^{+}\left(z q^{2 m-1}\right) \otimes \cdots \cdots \otimes \varphi\left(z q^{m-1 / 2}\right) x^{+}\left(z q^{m+1}\right) \otimes x\left(z q^{m}\right) .
\end{gathered}
$$

Proof. From the comultiplication formula, on $\otimes^{m+1} F$ we have

$$
\begin{gathered}
(1 \otimes 1 \otimes \ldots \otimes \Delta) \ldots(1 \otimes \Delta)(\Delta)\left(\left(x^{+}\right)(z)\right)= \\
x^{+}(z) \otimes 1 \ldots \otimes 1+\cdots+\varphi\left(z q^{1 / 2}\right) \otimes \varphi\left(z q^{3 / 2}\right) \otimes \ldots \otimes x^{+}\left(z q^{l}\right) \otimes \\
1 \ldots \otimes 1+\ldots+\varphi\left(z q^{1 / 2}\right) \otimes \varphi\left(z q^{3 / 2}\right) \otimes \ldots \otimes \varphi\left(z q^{m-1 / 2}\right) \otimes x^{+}\left(z q^{m}\right)= \\
\sum_{i=1}^{m} X_{i}^{+m}(z) .
\end{gathered}
$$

Let

$$
f_{a_{1}, \cdots a_{m+1}}^{m}\left(z_{1}, \ldots, z_{m+1}\right)=X_{a_{1}}^{+m}\left(z_{1}\right) \times \cdots X_{a_{m+1}}^{+m}\left(z_{m+1}\right) .
$$

First, we know that there are no poles at $z_{i} / z_{i+1}=q^{2}$ (Theorem 2.1). Let $0<i$ $<j \leq m+1$

$$
\begin{gathered}
\left(\varphi\left(z q^{1 / 2}\right) \otimes \varphi\left(z q^{3 / 2}\right) \otimes \ldots \otimes x^{+}\left(z q^{i}\right) \otimes 1 \ldots \otimes 1\right) \\
\left(\varphi\left(w q^{1 / 2}\right) \otimes \varphi\left(w q^{3 / 2}\right) \otimes \ldots \otimes x^{+}\left(w q^{j}\right) \otimes 1 \ldots \otimes 1\right)= \\
\varphi\left(z q^{1 / 2}\right) \varphi\left(w q^{1 / 2}\right) \otimes \varphi\left(z q^{3 / 2}\right) \varphi\left(w q^{3 / 2}\right) \otimes \ldots
\end{gathered}
$$

$\otimes: x^{+}\left(z q^{i-1}\right) \varphi\left(w q^{i-1 / 2}\right): \otimes \varphi\left(w q^{i+1 / 2}\right) \otimes \ldots x^{+}\left(w q^{j}\right) \otimes \ldots \otimes 1 \frac{\left(\frac{w}{z}-q^{2}\right)}{\left(\frac{w}{z} q^{2}\right)-1}$.

Thus know that if there is any $i$, such that $a_{i}<a_{i+1}$, then the correlation functions of $f_{a_{1}, \cdots a_{m+2}}^{m}\left(z_{1}, \ldots, z_{m+2}\right)$ are zero at $z_{i} / z_{i+1}=q^{2}$. That means the elements that are possibly not zero have the property that $a_{i} \leq a_{j}$, if $i>j$. Because $x^{+}(z) x^{+}\left(z q^{2}\right)$ is zero, we have that the elements that are possibly not 
zero have the property that $a_{i}<a_{j}$, if $i>j$. Therefore we finish the proof.

Let $x^{+m}(z)=\Delta^{m}\left(x^{+}(z)\right) \Delta^{m}\left(x^{+}\left(z q^{2}\right)\right) \cdots \Delta^{m}\left(x^{+}\left(z q^{(2 m)}\right)\right)$.

\section{Theorem 2.8 .}

$$
\begin{gathered}
x^{+m}\left(z q^{2}\right)=\varphi^{-1}\left(z q^{1 / 2}\right) \otimes \varphi^{-1}\left(z q^{3 / 2}\right) \otimes \ldots \varphi^{-1}\left(z q^{\frac{1}{2}+m}\right) x^{+m}(z) \\
\psi\left(z q^{\frac{1}{2}+2 m+1}\right) \otimes \ldots . \otimes \psi\left(z q^{\frac{1}{2}+1+m}\right)= \\
(1 \otimes 1 \otimes \ldots \otimes \Delta) \ldots(1 \otimes \Delta)(\Delta) \varphi^{-1}\left(z q^{m / 2+1 / 2}\right) x^{+m}(z) \\
(1 \otimes 1 \otimes \ldots \otimes \Delta) \ldots(1 \otimes \Delta)(\Delta) \psi\left(z q^{(m+1) 3 / 2}\right) .
\end{gathered}
$$

Proof.

$$
\begin{gathered}
x^{+m}\left(z q^{2}\right)=\varphi\left(z q^{2} q^{1 / 2}\right) \varphi\left(z q^{2} q^{1 / 2+2}\right) \cdots \varphi\left(z q^{2} q^{1 / 2+2 m-2}\right) \\
x^{+}\left(z q^{2} q^{2 m}\right) \otimes \varphi\left(z q^{2} q^{3 / 2}\right) \varphi\left(z q^{2} q^{3 / 2+2}\right) \cdots \varphi\left(z q^{2} q^{3 / 2+2 m-4} x^{+}\left(z q^{2} q^{2 m-1}\right) \otimes\right. \\
\cdots \cdots \otimes\left(z q^{2} q^{m-1 / 2}\right) x^{+}\left(z q^{2} q^{m+1}\right) \otimes x^{+}\left(z q^{2} q^{m}\right)= \\
\varphi\left(z q^{2} q^{1 / 2}\right) \varphi\left(z q^{2} q^{1 / 2+2}\right) \cdots \varphi\left(z q^{2} q^{1 / 2+2 m-2}\right) \varphi^{-1}\left(z q^{-\frac{1}{2}+1+2 m}\right) x^{+}\left(z q^{2 m}\right) \psi\left(z q^{\frac{1}{2}+1+2 m}\right) \otimes \\
\varphi\left(z q^{2} q^{3 / 2}\right) \varphi\left(z q^{2} q^{3 / 2+2}\right) \cdots \varphi\left(z q ^ { 2 } q ^ { 3 / 2 + 2 m - 4 } \varphi ^ { - 1 } \left(z q^{-\frac{1}{2}+2 m} x+\left(z q^{2 m-1}\right) \psi\left(z q^{\frac{1}{2}+2 m}\right) \otimes \ldots\right.\right. \\
\otimes \varphi\left(z q^{2} q^{m-1 / 2}\right) \varphi^{-1}\left(z q^{-\frac{1}{2}+1+m+1}\right) x^{+}\left(z q^{m+1}\right) \psi\left(z q^{\frac{1}{2}+1+m+1}\right) \otimes \varphi^{-1}\left(z q^{-\frac{1}{2}+1+m}\right) \otimes \\
x^{+}\left(z q^{m}\right) \psi\left(z q^{\frac{1}{2}+m+1}\right)=\varphi^{-1}\left(z q^{1 / 2}\right) \otimes \varphi^{-1}\left(z q^{3 / 2}\right) \otimes \ldots \varphi^{-1}\left(z q^{\frac{1}{2}+m}\right) x^{+m}(z) \times \\
\phi\left(z q^{\frac{1}{2}+2 m+2}\right) \otimes \ldots . \cdots \otimes\left(z q^{\frac{1}{2}+1+m}\right) .
\end{gathered}
$$

On the module $\otimes^{m+1} \underset{F}{\mathrm{~F}}$, from the comultiplication formula, we have

$$
\begin{gathered}
\Delta^{m}\left(a_{ \pm n}\right)=(1 \otimes 1 \otimes \ldots \otimes \Delta) \ldots(1 \otimes \Delta)(\Delta) a_{ \pm n}= \\
q^{(m) n / 2} a_{ \pm n} \otimes 1 \ldots \otimes 1+1 \otimes q^{(m-2) n / 2} a_{ \pm n} \otimes 1 \ldots \otimes 1+\ldots+ \\
1 \otimes \ldots \otimes 1 \otimes q^{-(m) n / 2} a_{ \pm n} .
\end{gathered}
$$

Then

$$
\left[\Delta^{m}\left(a_{n}\right), \Delta^{m}\left(a_{-n}\right)\right]=[2 n][(m+1) n] / n .
$$

From the theorem above, we have that

$$
\begin{aligned}
& x^{+m}(z)=\exp \left[\sum_{k>0} \frac{\Delta^{m}\left(a_{-k}\right)}{[k]} q^{m / 2-1 / 2} z^{k}\right] \exp \left[-\sum_{k>0} \frac{\Delta^{m}\left(a_{k}\right)}{[k]} q^{-3 m / 2-1 / 2} z^{-k}\right] \times \\
& \left(e^{\alpha} \otimes e^{\alpha} \ldots \ldots \otimes e^{\alpha}\right)\left(z^{\partial_{\alpha}+1} \otimes \ldots \otimes z^{\partial \alpha+1}\right)\left(q^{m \partial \alpha} \otimes \ldots \otimes q^{m \partial \alpha}\right) q^{(1+m) m / 2} .
\end{aligned}
$$

Corollary 2.9. $x^{+m}(z)$ is an vertex operator.

Similarly we can derive corresponding results for $x^{-}(z)$. 
Lemma 2.10. On $\otimes^{m+1} F$, let $\Delta^{m}\left(x^{-}(z)\right)=(1 \otimes 1 \otimes \ldots \otimes \Delta) \ldots(1 \otimes \Delta)(\Delta)$ $\left(\left(x^{-}\right)(z)\right)$, then

$$
\begin{gathered}
\Delta^{m}\left(x^{-}\left(z q^{(2 m)}\right)\right) \Delta^{m}\left(x^{-}\left(z q^{2 m-1}\right)\right) \cdots \Delta^{m}\left(x^{-}(z)\right)= \\
x^{-}\left(z q^{m}\right) \otimes x^{-}\left(z q^{m+1}\right) \psi\left(z q^{3 / 2+2 m-2}\right) \otimes \ldots \otimes \\
x^{-}\left(z q^{2 m-1}\right) \psi\left(z q^{3 / 2+2 m-4}\right) \cdots \psi\left(z q^{3 / 2+2}\right) \psi\left(z q^{3 / 2}\right) \otimes \\
x^{-}\left(z q^{2 m}\right) \psi\left(z q^{1 / 2+2 m-2}\right) \cdots \psi\left(z q^{1 / 2+2}\right) \psi\left(z q^{1 / 2}\right) .
\end{gathered}
$$

Let $x^{-m}(z)=\Delta^{m}\left(x^{-}\left(z q^{(2 m)}\right)\right) \Delta^{m}\left(x^{-}\left(z q^{2 m-2}\right)\right) \cdots \Delta^{m}\left(x^{-}(z)\right)$.

Theorem 2.11.

$$
\begin{gathered}
x^{-m}\left(z q^{2}\right)=\varphi\left(z q^{\frac{1}{2}+1+m}\right) \otimes \ldots \otimes \varphi\left(z q^{\frac{1}{2}+2 m+1}\right) x^{-m}(z) \\
\psi(z)^{-1}\left(z q^{\frac{1}{2}+m} \otimes \ldots \psi^{-1}\left(z q^{3 / 2}\right) \otimes \psi^{-1}\left(z q^{1 / 2}\right)=\right. \\
(1 \otimes 1 \otimes \ldots \otimes \Delta) \ldots(1 \otimes \Delta)(\Delta) \varphi\left(z q^{-(m+1) 3 / 2}\right) x^{-m}(z) \\
(1 \otimes 1 \otimes \ldots \otimes \Delta) \ldots(1 \otimes \Delta)(\Delta) \psi^{-1}\left(z q^{-m / 2+1 / 2}\right) .
\end{gathered}
$$

Then, from the theorem above, we have that

$$
\begin{aligned}
x^{-m}(z)= & \exp \left[-\sum_{k>0} \frac{\Delta^{m}\left(a_{-k}\right)}{[k]} q^{(1+3 m / 2) k} z^{k}\right] \exp \left[\sum_{k>0} \frac{\Delta^{m}\left(a_{k}\right)}{[k]} q^{(1-m) / 2)^{k}} z^{-k}\right] \\
& \left(e^{-\alpha} \otimes e^{-\alpha} \ldots \ldots \otimes e^{-\alpha}\right)\left(z^{-\partial a l p h a+1} \otimes \ldots . z^{-\partial a l p h a+1}\right) \\
& \left(q^{-m \partial a l p h a} \otimes \ldots \otimes q^{-m \partial a l p h a}\right) q^{(1+m) m / 2}
\end{aligned}
$$

Corollary 2.12. $x^{-m}(z)$ is an vertex operator.

\section{§3. Quantum Parafermions}

In this section, we will derive quantum parafermion and explain the parafermionic construction of integrable modules of $U_{q}(\hat{\mathfrak{g} l}(2))$ following the line of the work of Lepowsky and Wilson [LW1] [LW2]. This type of construction for the classical case was also given in [FZ] from a different point of view.

On the module $\otimes^{m+1} \mathrm{~F}$, we have that

$$
\begin{gathered}
(1 \otimes 1 \otimes \ldots \otimes \Delta) \ldots(1 \otimes \Delta)(\Delta)\left(\left(x^{+}\right)(z)\right)= \\
x^{+}(z) \otimes 1 \ldots \otimes 1+\ldots+\varphi\left(z q^{1 / 2}\right) \otimes \varphi\left(z q^{3 / 2}\right) \otimes \ldots \otimes x^{+}\left(z q^{l}\right) \otimes 1 \ldots \otimes 1 \\
+\ldots+\varphi\left(z q^{1 / 2}\right) \otimes \varphi\left(z q^{3 / 2}\right) \otimes \ldots \otimes \varphi\left(z q^{m-1 / 2}\right) \otimes x^{+}\left(z q^{m}\right) .
\end{gathered}
$$

So the $l+1$-th term $X_{l}^{+m}$ is 


$$
\begin{gathered}
\varphi\left(z q^{1 / 2}\right) \otimes \varphi\left(z q^{3 / 2}\right) \otimes \ldots \otimes \varphi\left(z q^{l-1 / 2}\right) \otimes x^{+}\left(z q^{l}\right) \otimes 1 \ldots \otimes 1= \\
\exp \left[-\left(q-q^{-1}\right) \sum_{k>0} a_{-k} q^{k / 2} z^{k}\right] q^{-\partial \alpha} \otimes \exp \left[-\left(q-q^{-1}\right) \sum_{k>0} a_{-k} q^{3 k / 2} z^{k}\right] q^{-\partial \alpha} \otimes \\
\ldots \otimes \exp \left[-\left(q-q^{-1}\right) \sum_{k>0} a_{-k} q^{k(l-1 / 2)} z^{k}\right] q^{-\partial \alpha} \otimes \exp \left[\sum_{k>0} \frac{a_{-k}}{[k]} q^{-\frac{1}{2} k} q^{l k} z^{k}\right] \\
\exp \left[-\sum_{k>0} \frac{a_{k}}{[k]} q^{-\frac{1}{2} k} q^{-l k} z^{-k}\right] e^{\alpha} z^{\partial \alpha+1} q^{l o} q^{l}= \\
\exp \left[-\left(q-q^{-1}\right) \sum_{k>0} z^{k}\left(a_{-k} q^{k / 2} \otimes 1 \ldots \otimes 1+1 \otimes a_{-k} q^{3 k / 2} \otimes \ldots \otimes 1+\ldots+\right.\right. \\
\left.\left.1 \otimes \ldots \otimes a_{-k} q^{l-1 / 2} \otimes 1 \ldots \otimes 1\right)+1 \otimes \ldots \otimes 1 \otimes \frac{a_{-k}}{[k]} q^{-\frac{1}{2} k} q^{l k} \otimes 1 \ldots \otimes 1\right] \\
\exp \left[1 \otimes \ldots \otimes 1-\sum_{k>0} \frac{a_{k}}{[k]} q^{-\frac{1}{2} k} q^{-l k} \otimes 1 \ldots \otimes 1\right]\left(1 \otimes \ldots \otimes 1 \otimes e^{\alpha} \otimes 1 \ldots \otimes 1\right) \\
\left.q^{-\partial \alpha} \otimes \ldots \otimes q^{-\partial \alpha} \otimes z^{\partial \alpha+1} q^{l \partial \alpha} \otimes 1 \ldots \otimes 1\right) q^{l} .
\end{gathered}
$$

\section{Lemma 3.1.}

$\left[\Delta^{m}\left(a_{k}\right),-\left(q-q^{-1}\right)\left(a_{-k} q^{k / 2} \otimes 1 \ldots \otimes 1+1 \otimes a_{-k} q^{3 k / 2} \otimes \ldots \otimes 1+\ldots+1 \otimes\right.\right.$

$\left.\left.\ldots \otimes 1 \otimes a_{-k} q^{l-1 / 2} \otimes 1 \ldots \otimes 1\right)+1 \otimes \ldots \otimes 1 \otimes \frac{a_{-k}}{[k]} q^{-\frac{1}{2} k} q^{l k} \otimes 1 \ldots \otimes 1\right]=$

$$
\begin{gathered}
{[2 k] q^{-k(m+1) / 2}} \\
{\left[1 \otimes \ldots \otimes 1-\sum_{k>0} \frac{a_{k}}{[k]} q^{-\frac{1}{2} k} q^{-l k} \otimes 1 \ldots \otimes 1, \Delta^{m}\left(a_{-k}\right)\right]=} \\
-q^{((-m-1) / 2+l) k}[2 k] / n\left(q^{-\frac{1}{2} k} q^{-l k}\right)= \\
-q^{-k(m+1) / 2}[2 k] / n .
\end{gathered}
$$

Proof.

$$
\begin{gathered}
{\left[\Delta^{m}\left(a_{k}\right),-\left(q-q^{-1}\right)\left(a_{-k} q^{k / 2} \otimes 1 \ldots \otimes 1+1 \otimes a_{-k} q^{3 k / 2} \otimes \ldots \otimes 1+\ldots+1 \otimes\right.\right.} \\
\left.\left.\ldots \otimes 1 \otimes a_{-k} q^{l-1 / 2} \otimes 1 \ldots \otimes 1\right)+1 \otimes \ldots \otimes 1 \otimes \frac{a_{-k}}{[k]} q^{-\frac{1}{2} k} q^{l k} \otimes 1 \ldots \otimes 1\right]= \\
{[2 k][k] / n\left(q^{k / 2} q^{k(-m) / 2}+q^{3 / 2 k} q^{k(-m+2) / 2}+\right.} \\
\left.\left.\ldots+q^{(l-1 / 2) k} q^{((-m) / 2+l-1) k}\right)\left(-q+q^{-1}\right)+q^{-k / 2} q^{l k} q^{((-m) / 2+l) k} /[k]\right)= \\
{[2 k][k] / n\left(q^{k / 2} q^{k(-m) / 2}\left(1+\ldots+q^{2(l-1) k}\right)+q^{-k / 2} q^{l k} q^{((-m) / 2+l) k} /[k]\right)=} \\
\left(-q+q^{-1}\right)[2 k][k] / n\left(q^{k / 2} q^{k(-m) / 2}\left(1-q^{2 l k} /\left(1-q^{2 k}\right)+q^{-k / 2} q^{l k} q^{((-m) / 2+l) k} /[k]\right)=\right. \\
{[2 k] q^{-k / 2} q^{k(-m) / 2} / n=[2 k] q^{-k(m+1) / 2} .}
\end{gathered}
$$

From the comultiplication formula, we have

$$
\Delta^{m}\left(x^{-}(z)\right)=(1 \otimes 1 \otimes \ldots \otimes \Delta) \ldots(1 \otimes \Delta)(\Delta)\left(\left(x^{-}\right)(z)\right)=
$$




$$
\begin{aligned}
& 1 \otimes \ldots \otimes 1 \otimes x^{-}(z)+1 \otimes \ldots \otimes 1 \otimes x^{-}\left(z q^{1}\right) \otimes \psi\left(z q^{l-\frac{1}{2}}\right) \otimes \ldots+ \\
& \ldots+x^{-}\left(z q^{m}\right) \otimes \psi\left(z q^{m-\frac{1}{2}}\right) \otimes \ldots \otimes \psi\left(z q^{\frac{1}{2}}\right)= \\
& \sum_{i=1}^{m} X_{i}^{-m}(z) \\
& X_{i}^{-m}(z)=1 \otimes \ldots \otimes 1 \otimes x^{-}\left(z q^{i-1}\right) \otimes \psi\left(z q^{i-1-\frac{1}{2}}\right)= \\
& 1 \otimes \ldots \otimes \exp \left[-\sum_{k>0} \frac{a_{-k}}{[k]} q^{(i-1) k+\frac{1}{2} k} z^{k}\right] \\
& \exp \left[\sum_{k>0} \frac{a_{k}}{[k]} q^{-(i-1) k+\frac{1}{2} k} z^{-k}\right] e^{-\alpha} z^{-\partial_{\alpha}+1} q^{-(i-1) \partial \alpha} q^{(i-1)} \otimes \\
& q^{\partial a} \exp \left[( q - q ^ { - 1 } \sum _ { k > 0 } a _ { k } q ^ { ( i - 1 ) k - \frac { 1 } { 2 } k } z ^ { - k } ] \otimes \ldots \otimes q ^ { \partial \alpha } \operatorname { e x p } \left[\left(q-q^{-1} \sum_{k>0} a_{k} q^{\frac{1}{2} k} z^{-k}\right]=\right.\right. \\
& \left.\exp \left[1 \otimes \ldots \otimes-\sum_{k>0} \frac{a_{-k}}{[k]} q^{(i-1) k+\frac{1}{2} k} z^{k}\right] \otimes \ldots \otimes 1\right] \times \\
& \exp \left[1 \otimes 1 \ldots \otimes 1 \sum_{k>0} \frac{a_{k}}{[k]} q^{-(i-1) k+\frac{1}{2} k} z^{-k} \otimes 1 \ldots \otimes 1\right. \\
& +\ldots 1 \otimes \ldots 1 \otimes\left(q-q^{-1}\right) \sum_{k>0} a_{k} q^{(i-1) k-\frac{1}{2} k} z^{-k} \otimes \\
& \left.1 \ldots \otimes 1 \ldots+\ldots+1 \otimes \ldots \otimes 1\left(q-q^{-1}\right) \sum_{k>0} a_{k} q^{\frac{1}{2} k} z^{-k} \otimes 1 \ldots \otimes 1\right] \times \\
& \left(1 \otimes \ldots \otimes 1 \otimes e^{-\alpha} \otimes 1 \ldots \otimes 1\right)\left(1 \otimes \ldots 1 \otimes z^{-\partial_{\alpha}+1} q^{-(i-1) \partial \alpha} \otimes q^{\partial \alpha} \otimes \ldots \otimes q^{\partial \alpha} q^{(i-1)} .\right.
\end{aligned}
$$

\section{Lemma 3.2.}

$$
\left.\left[\Delta^{m}\left(a_{k}\right), 1 \otimes \ldots \otimes-\frac{a_{-k}}{[k]} q^{(i-1) k+\frac{1}{2} k}\right] \otimes 1 \ldots \otimes 1\right]=-[2 k] / n\left(q^{m k / 2+k / 2}\right)
$$

$\left[1 \otimes 1 \ldots \otimes 1 \frac{a_{k}}{[k]} q^{-(i-1) k+\frac{1}{2} k} z^{-k} \otimes 1 \ldots \otimes 1+1 \otimes \ldots 1 \otimes\left(q-q^{-1}\right) a_{k} q^{(i-1) k-\frac{1}{2} k} z^{-k}\right.$

$\left.\otimes 1 \ldots \otimes 1 \ldots+\ldots+1 \otimes \ldots \otimes 1\left(q-q^{-1}\right) \sum_{k>0} a_{k} q^{\frac{1}{2} k} z^{-k} \otimes 1 \ldots \otimes 1, \Delta^{m}\left(a_{-k}\right)\right]=$

$$
[2 k] / n\left(q^{m k / 2+k / 2}\right) \text {. }
$$

Let

$$
\begin{gathered}
V( \pm m, z)=\exp \left[\frac{\Delta^{m}\left(a_{-k}\right)}{[(m+1) k]} q^{\mp(m+1) k / 2} z^{k}\right] \exp \left[\frac{\Delta^{m}\left(a_{k}\right)}{[(m+1) k]} q^{\mp(m+1) k / 2} z^{-k}\right] \times \\
\left(e^{ \pm \alpha /(m+1)} \otimes \ldots \ldots \otimes e^{ \pm \alpha /(m+1)}\right)\left(z^{ \pm \partial \alpha /(m+1)} \otimes \ldots \ldots \otimes z^{ \pm \partial \alpha /(m+1)}\right) z .
\end{gathered}
$$

\section{Lemma 3.3.}

$$
V( \pm m, z) V( \pm m, w)
$$




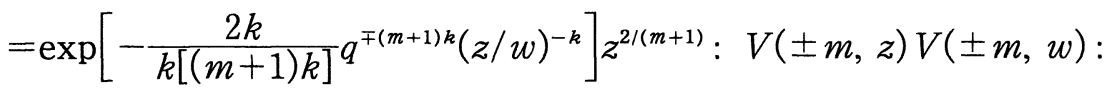

$$
\begin{aligned}
& V( \pm m, z) V(\mp m, w) \\
& =\exp \left[\frac{2 k}{k[(m+1) k]}(z / w)^{-k}\right] z^{-2 /(m+1)} e^{-2 \pi i /(m+1)}: V( \pm m, z) V(\mp m, w) \text { : }
\end{aligned}
$$

We will denote $\exp \left[-\frac{2 k}{[(m+1) k]} q^{\mp(m+1) k}(z / w)^{-k}\right] z^{2 /(m+1)}$ by $f^{ \pm}(w, z)$ and $\exp \left[\frac{2 k}{[(m+1) k]}(z / w)^{-k}\right] z^{-2 /(m+1)}$ by $p(w, z)$.

Let $X^{ \pm m}(z)=V( \pm m, z) \phi^{ \pm m}(z)$ and $X_{i}^{ \pm m}(z)=V( \pm m, z) \phi_{i}^{ \pm m}(z)$. We have that $\phi^{ \pm m}(z)=\sum \phi_{i}^{ \pm m}(z)$.

Proposition 3.4. On the space $\otimes^{m+1} F$,

$$
\begin{aligned}
& \phi^{ \pm m}(z) \Delta^{m}(\varphi(w))=\Delta^{m}(\varphi(w)) \phi^{ \pm m}(z), \\
& \phi^{ \pm m}(z) \Delta^{m}(\varphi(w))=\Delta^{m}(\varphi(w)) \phi^{ \pm m}(z), \\
& \phi^{ \pm m}(z) \Delta^{m}(\psi(w))=\Delta^{m}(\psi(w)) \phi^{ \pm m}(z), \\
& \phi^{ \pm m}(z) \Delta^{m}(\psi(w))=\Delta^{m}(\psi(w)) \phi^{ \pm m}(z) .
\end{aligned}
$$

As the commutants to $\Delta^{m}(\varphi(w))$ and $\Delta^{m}(\phi(w)), \phi^{ \pm m}(z)$ degenerate into the classical parafermions respectively [LW1] [LW2] [FZ]. Thus $\phi^{ \pm m}(z)$ gives us the quantum parafermions.

Proposition 3.5. On the space $\otimes^{m+1} F$,

$$
\begin{gathered}
: \phi_{m+1}^{+m}(z) \phi_{m}^{+m}\left(z q^{2}\right) \ldots \phi_{1}^{+m}\left(z q^{2 m}\right):=1 \\
: \phi_{1}^{-m}(z) \phi_{2}^{-m}\left(z q^{-2}\right) \ldots \phi_{m+1}^{-m}\left(z q^{-2 m}\right):=1
\end{gathered}
$$

This is a quantum version of a classical relations [LW2] [FZ], which comply with the results in $[\mathrm{DM}]$.

From the calculation above, we can easily write down the commutation relations between $\phi_{i}^{ \pm m}(z)$ and $\phi_{i}^{ \pm m}(w)$ and the commutation relations between $\phi_{i}^{ \pm m}(z)$ and $\phi_{i}^{\mp m}(w)$.

Lemma 3.6. If $i<j$,

$$
\begin{aligned}
X_{i}^{+m}(z) X_{j}^{+m}(w) & =\frac{\left(\frac{w}{z}-q^{2}\right)}{\left(\frac{w q^{2}}{z}-1\right)} X_{j}^{+m}(w) X_{i}^{+m}(z)=g(w / z)^{-1} X_{j}^{+m}(w) X_{i}^{+m}(z) \\
X_{j}^{+m}(z) X_{i}^{+m}(w) & =\frac{\left(\frac{z q^{2}}{w}-1\right)}{\left(\frac{z}{w}-q^{2}\right)} X_{i}^{+m}(w) X_{j}^{+m}(z)=g(z / w) X_{i}^{+m}(w) X_{j}^{+m}(z)
\end{aligned}
$$




$$
\begin{gathered}
X_{j}^{-m}(z) X_{i}^{-m}(w)=\frac{\left(\frac{z q^{2}}{w}-1\right)}{\left(\frac{z}{w}-q^{2}\right)} X_{i}^{-m}(w) X_{j}^{-m}(z)=g(z / w) X_{i}^{-m}(w) X_{j}^{-m}(z) \\
X_{i}^{-m}(z) X_{j}^{-m}(w)=\frac{\left(\frac{w}{z}-q^{2}\right)}{\left(\frac{w q^{2}}{z}-1\right)} X_{j}^{-m}(w) X_{i}^{-m}(z)=g(w / z)^{-1} X_{j}^{-m}(w) X_{i}^{-m}(z) \\
X_{i}^{+m}(z) X_{j}^{\mp m}(w)=X_{j}^{\mp m}(w) X_{i}^{+m}(z) ; \\
X_{i}^{+m}(z) X_{i}^{+m}(w)=z^{2} \frac{\left(1-\frac{w}{z}\right)}{\left(1-\frac{w}{z q^{2}}\right)}: X_{i}^{+m}(w) X_{i}^{+m}(z) ; \\
X_{i}^{-m}(z) X_{i}^{-m}(w)=z^{2}\left(1-\frac{w}{z}\right)\left(1-\frac{w q^{2}}{z}\right): X_{i}^{-m}(z) X_{i}^{-m}(w): \\
X_{i}^{+m}(z) X_{i}^{-m}(w)=z^{-2} \frac{1}{\left(1-\frac{w}{z q}\right)\left(1-\frac{w}{z q^{-1}}\right)}: X_{i}^{-m}(w) X_{i}^{+m}(z) ; \\
X_{i}^{-m}(z) X_{i}^{+m}(w)=w^{-2} \frac{1}{\left(1-\frac{w}{z q}\right)\left(1-\frac{w}{z q^{-1}}\right)}: X_{i}^{-m}(z) X_{i}^{+m}(w) ;
\end{gathered}
$$

Lemma 3.7. If $i<j$,

$$
\begin{gathered}
f^{+}(w, z) \phi_{i}^{+m}(z) \phi_{j}^{+m}(w)=f^{+}(z, w) \frac{\left(\frac{w}{z}-q^{2}\right)}{\left(\frac{w q^{2}}{z}-1\right)} \phi_{j}^{+m}(w) \phi_{i}^{+m}(z)= \\
f^{+}(z, w) g(w / z)^{-1} \phi_{j}^{+m}(w) \phi_{i}^{+m}(z), \\
f^{+}(w, z) \phi_{j}^{+m}(z) \phi_{i}^{+m}(w)=f^{+}(z, w) \frac{\left(\frac{z q^{2}}{w}-1\right)}{\left(\frac{z}{w}-q^{2}\right)} \phi_{i}^{+m}(w) \phi_{j}^{+m}(z)= \\
f^{+}(z, w) g(z / w) \phi_{i}^{+m}(w) \phi_{j}^{+m}(z), \\
f^{-}(w, z) \phi_{j}^{-m}(z) \phi_{i}^{-m}(w)=f^{-}(z, w) \frac{\left(\frac{z q^{2}}{w}-1\right)}{\left(\frac{z}{w}-q^{2}\right)} \phi_{i}^{-m}(w) \phi_{j}^{-m}(z)= \\
f^{-}(w, z) \phi_{i}^{-m}(z) \phi_{j}^{-m}(w)=f^{-}(z, w) \frac{\left(\frac{w}{z}-q^{2}\right)}{\left(\frac{w q^{2}}{z}-1\right)} \phi_{j}^{-m}(w) \phi_{i}^{-m}(z)= \\
f^{-}(z, w) g(w / z)^{-1} \phi_{j}^{-m}(w) \phi_{i}^{-m}(z), \\
p^{ \pm}(w, z) \phi_{i}^{ \pm m}(z) \phi_{j}^{\mp m}(w)=p^{ \pm}(z, w) \phi_{j}^{\mp m}(w) \phi_{i}^{ \pm m}(z)
\end{gathered}
$$




$$
\begin{gathered}
\phi_{i}^{+m}(z) \phi_{i}^{+m}(w)=f^{+}(w, z) z^{2} \frac{\left(1-\frac{w}{z}\right)}{\left(\frac{1}{-} w z q^{2}-1\right)}: \phi_{i}^{+m}(w) \phi_{i}^{+m}(z) ; \\
f^{+}(w, z) \phi_{i}^{-m}(z) \phi_{i}^{-m}(w)=f^{-}(w, z) z^{2}\left(1-\frac{w}{z}\right)\left(1-\frac{w q^{2}}{z}\right): \phi_{i}^{-m}(z) \phi_{i}^{-m}(w): . \\
\phi_{i}^{+m}(z) \phi_{i}^{-m}(w)=p(w, z) z^{-2} \frac{1}{\left(1-\frac{w}{z q}\right)\left(\frac{1}{-} w z q^{-1}\right)}: \phi_{i}^{-m}(w) \phi_{i}^{+m}(z):, \\
\phi_{i}^{-m}(z) \phi_{i}^{+m}(w)=p(w, z) w^{-2} \frac{1}{\left(1-\frac{w}{z q}\right)\left(\frac{1}{-} w z q^{-1}\right)}: \phi_{i}^{-m}(z) \phi_{i}^{+m}(w): .
\end{gathered}
$$

Theorem 3.8.

$$
\begin{gathered}
{\left[p(w, z) \phi^{+m}(z) \phi^{-m}(w)-p(z, w) \phi^{-m}(w) \phi^{+m}(z)\right]} \\
=\frac{1}{q-q^{-1}}\left(\delta\left(z / w q^{m+1}\right)-\left(\delta\left(z / w q^{-(m+1)}\right),\right.\right. \\
f^{ \pm}(w, z)\left(z-w q^{ \pm 2}\right) \phi^{ \pm(z) m} \phi^{ \pm m}(w)=f^{\mp}(z, w)\left(z q^{ \pm 2}-w\right) \phi^{ \pm m}(w) \phi^{ \pm m}(z) .
\end{gathered}
$$

\section{Corollary 3.9.}

$$
\begin{gathered}
\phi^{+1}(z)=\phi^{-1}(z), \\
\phi_{i}^{+1}(z)=\phi_{i}^{-1}(z), i=1,2 ; \\
\phi_{i}^{ \pm 1}(z) \phi_{i}^{ \pm}(w)=-\phi_{i}^{ \pm 1}(w) \phi_{i}^{ \pm 1}(z), i=1,2 ; \\
\left\{\phi_{i}^{+1}(z), \phi_{i}^{-1}(w)\right\}=\delta\left(q^{-2} z / w\right) .
\end{gathered}
$$

We can see that the quantum fermion $\phi^{+1}(z)$ is basically the same as the classical fermion, but with certain shifts.

Lemma 3.10. On the module $\otimes^{m+1} F, N<m$,

$$
\begin{gathered}
\Delta^{m}\left(x^{+}(z)\right) \Delta^{m}\left(x^{+}\left(z q^{2}\right)\right) \ldots \Delta^{m}\left(x^{+}\left(z q^{2 N-2}\right)\right) \Delta^{m}\left(x^{+}\left(z q^{2 N}\right)\right)= \\
\sum_{i^{i_{1}>i_{2} \cdots>i_{N+1}}} X_{i_{1}}^{+m}(z) \ldots X_{i_{N+1}}^{+m}\left(z q^{2 N}\right) ; \\
\Delta^{m}\left(x^{-}\left(z q^{2 N}\right)\right) \Delta^{m}\left(x^{-}\left(z q^{2 N-2}\right)\right) \ldots \Delta^{m}\left(x^{-}\left(z q^{2}\right)\right) \Delta^{m}\left(x^{-}(z)\right)= \\
\sum_{j_{1}<j_{2} \cdots<j_{N+1}} X_{j_{1}}^{-m}\left(z q^{2 n}\right) \ldots X_{N_{N+1}}^{-m}(z) .
\end{gathered}
$$

We can prove it with the same method as in Lemma 2.7.

Lemma 3.11. Let $0 \leq N<m, i_{1}>i_{2} \ldots>i_{N+1}, j_{1}<j_{2} \ldots<j_{m-N}$ and the set $\left\{i_{1}, \ldots, i_{N+1}, j_{1}, \ldots, j_{m-N}\right\}$ is the set $\{1,2,3, \ldots, m+1\}$. On the module $\otimes^{m+1} F$, let 


$$
\begin{gathered}
X_{i_{1}}^{+m}(z) \ldots X_{i_{N+1}}^{+m}\left(z q^{2 N}\right)= \\
: V(+m, z) V\left(+m, z q^{2}\right) \ldots V\left(+m, z q^{2 N}\right): \phi_{i_{1}>i_{2} \cdots>i_{N+1}}^{+m}(z), \\
X_{j_{1}}^{-m}\left(z q^{2(m-N-1)}\right) \ldots X_{m_{-N+1}}^{-m}(z)= \\
: V(-m, z) V\left(-m, z q^{2}\right) \ldots V\left(-m, z q^{2(m-N-1)}\right): \phi_{j_{1}<j_{2} \cdots<j m-N+1}^{-m}(z) .
\end{gathered}
$$

Then

$$
\phi_{i_{1}>i_{2} \cdots>i_{N+1}}^{+m}=\phi_{j_{1}<j_{2} \cdots<j m-N+1}^{-m}(z)
$$

Proof. Let

$$
\bar{x}(z)=\exp \left[\sum_{k>0} \frac{a_{-k}}{[k]} q^{\frac{1}{2} k} z^{k}\right] \exp \left[-\sum_{k>0} \frac{a_{k}}{[k]} q^{\frac{1}{2} k} z^{-k}\right] e^{\alpha} z^{\partial_{\alpha}-1}
$$

Then

$$
\begin{gathered}
\bar{x}\left(z q^{2}\right)=\varphi\left(z q^{3 / 2}\right)^{-1} \bar{x}(z) \psi\left(z q^{1 / 2}\right) \\
\bar{x}\left(z q^{-1}\right)=x^{+}(z) \psi\left(z q^{-1 / 2}\right)^{-1} q . \\
\varphi\left(z q^{1 / 2+l}\right) \varphi\left(z q^{1 / 2+2+l}\right) \cdots \varphi\left(z q^{1 / 2+2 m-l-2}\right) x^{+}\left(z q^{2 m-l}\right)= \\
\varphi\left(z q^{1 / 2+l}\right) \varphi\left(z q^{1 / 2+2+l}\right) \cdots \varphi\left(z q^{1 / 2+2 m-l-2}\right) x^{+}\left(z q^{2 m}\right) \psi\left(z q^{(2 m-l)+3 / 2}\right)^{-1} \cdots \\
\phi\left(z q^{2 m+3 / 2}\right)^{-1} \cdot . \\
\varphi\left(z q^{1 / 2+l}\right) \varphi\left(z q^{1 / 2+2+l}\right) \cdots \varphi\left(z q^{1 / 2+2 m-l-2}\right) x^{+}\left(z q^{2 m-l}\right)= \\
\varphi\left(z q^{1 / 2+l}\right) \varphi\left(z q^{1 / 2+2+l}\right) \cdots \varphi\left(z q^{1 / 2+2 m-l-2}\right) \bar{x}\left(z q^{2 m-l-1}\right) \psi\left(z q^{2 m-l-1 / 2}\right) q= \\
\varphi\left(z q^{1 / 2+l}\right) \varphi\left(z q^{1 / 2+2+l}\right) \cdots \varphi\left(z q^{1 / 2+2 m-l-2 k-2}\right) \bar{x}\left(z q^{2 m-l-2 k-1}\right) \psi\left(z q^{2 m-l-2 k-1 / 2}\right) \\
\cdots \psi\left(z q^{2 m-l-1-3 / 2}\right) \psi\left(z q^{2 m-l-1 / 2}\right) q .
\end{gathered}
$$

This gives us the proof.

Corollary 3.12. For $0 \leq N<m$, let

$$
\begin{gathered}
\phi^{+m, N}(z)=\sum \phi_{i_{1}>i_{2} \cdots>i_{N+1}}^{+m}(z) \\
\phi^{-m, m-N-1}(z)=\sum \phi_{i_{1}<i_{2} \cdots<i_{m-N+1}}^{-m}(z) .
\end{gathered}
$$

Then

$$
\phi^{+m, N}(z)=\phi^{-m, m-N-1}(z)
$$

Let $V^{-}( \pm m, z)$ be the vertex operators such that : $V^{-}( \pm m, z) V( \pm m, z)$ $:=1$.

\section{Corollary 3.13.}

$: V^{-}(+m, z) \ldots V^{-}\left(+m, z q^{2 N}\right): \Delta^{m}\left(x^{+}(z)\right) \ldots \Delta^{m}\left(x^{+}\left(z q^{2} N\right)\right)=$ 
: $V^{-}\left(-m, z q^{2 m-2 N}\right) \ldots V^{-}(-m, z): \Delta^{m}\left(x^{-}\left(z q^{2 m-2 N}\right)\right) \ldots \Delta^{m}\left(x^{-}(z)\right)$.

This generalizes the corresponding results in $[\mathrm{LP}]$.

Definition 3.1. Let

$$
\phi^{m, N}=\sum \phi_{i_{1}>i_{2} \cdots>i_{N+1}}^{+m}(z),
$$

for $N<m$. We call $\phi^{m, N}(z)$ quantum parafermions.

These quantum parafermions degenerate into the classical parafermions, when q goes to 1 .

With the results above, we can write the operator product expansion of these quantum parafermions and the commutation relations between those quantum parafermions. The operator product expanison is very complicated as it can been see from the commutation relations below.

\section{Propositin 3.14.}

$$
\begin{gathered}
\prod_{\substack{j=0,1 . . N \\
i=0,1 ., M}}^{\substack{i=0, \ldots, M \\
j=0,1 . . N}}\left(z q^{2 j}-q^{2} w q^{2 i}\right) f^{+}\left(w q^{2 i}, z q^{2 j}\right) \phi^{m, N}(z) \phi^{m, M}(w)= \\
\prod_{\substack{i=0,1 ., m-M-1 \\
j=0,1 . . m-N-1 \\
i=0,1 . ., m-M+1}}\left(z q^{2 j}-q^{-2} w q^{2 i}\right) f^{-}\left(z q^{2 j}, w q^{2 i}\right) \phi^{m, M}(w) \phi^{m, N}(z) ; \\
\left.\prod_{j=0,1 . . m-N+1}^{2 j}\right) \phi^{m, N}(z) \phi^{m, M}(w)=
\end{gathered}
$$

A version of quantum parafermion is given in $[\mathrm{J}][\mathrm{BV}]$, where only $\phi^{m, 0}(z)$ and $\phi^{m, m-1}(z)$ are given. With the Drinfeld comultiplication, we are able to follow the line of [LW1] [LW2] to derive all the integrable representation. This automatically leads us to the quantum parafermions characterized as the commutant to $\varphi(z)$ and $\phi(z)$. Clearly, we can use the operators $\phi^{ \pm m}(z)$ to derive $x^{ \pm}(z)$, by $V( \pm, z) \phi^{ \pm}(z)$, which gives parafermion constructions. Combining the results in $[\mathrm{DM}]$, we bascially derive all the corresponding structure corresponding to the results in [LW1] [LW2] concerning the structure of standard modules for the case of $\hat{\mathfrak{s} l}(2)$, which essentially prepares all the necessary conditions for the extension of the results in [FS] to the quantum cases. On the other hand, parafermions in the classical case have very important applications in the conformal field theory $[\mathrm{FZ}]$. Our $\phi^{m, N}(z)$ as deformed fermions should play an important role in formulating the theory of the quantization of the conformal field theory. The results in this paper can be extended to the other cases of quantum affine algebras in a straightforward way, which will gives us the structure of the standard modules of the quantum affine algebras like in [LW1] [LW2]. 


\section{Acknowledgement}

The authors would like to thank Tetsuji Miwa for useful discussions. J. D. is supported by the grant Reward research (A) 08740020 from the Ministry of Education of Japan. B. F. would like to thank RIMS for fhe hospitality throughout his stay, during which this work was done.

\section{References}

[BV] Bougourzi, A. H. and Vinet, L., On a boson-parafermionic realization of $U_{q}(\hat{\mathfrak{s} l}(2)), C R M$ -2201 , hep-th/9407062

[DF] Ding, J. and Frenkel, I. B., Isomorphism of two realizations of quantum affine algebra $U_{q}(\hat{g l} l(n)), C M P, 156$ (1993), 277-300.

[DI] Ding, J. and Iohara, K., Drinfeld comultiplication and vertex operators, RIMS-1091, Preprint.

[DM] Ding, J. and Miwa, T., Zeroes and poles of current operators and quantum integrable condition, RIMS-1092, Preprint.

[Dr1] Drinfeld, V. G., Hopf algebra and the quantum Yang-Baxter Equation, Dokl. Akad, Nauk. SSSR, 283 (1985), 1060-1064.

[Dr2] _ Quantum Groups, ICM Proceedings, New York, Berkeley, 1986, 798-820

[Dr3] N New realization of Yangian and quantum affine algebra, Soviet Math. Doklady, 36 (1988), 212-216.

[FS] Feigin, B. L. and Stoyanovsky, A. V., Quasi-particle models for the representations of Lie algebras and the geometry of the flag manifold, RIMS-942, Preprint.

[FZ] Fateev, V.A. and Zamolodchikov, A. B., Nonlocal(parafermion) current in two dimensional conformal quantam field theory and seal-dual critical points in $\mathbf{Z}_{N}$-symmetric statistical systems, Sov. Phys. JETP, 62(2) (1985), 215-225.

[J1] Jimbo, M., A $q$-difference analogue of $U(\mathrm{~g})$ and Yang-Baxter equation, Lett. Math. Phys., 10 (1985), 63-69.

[J] Jing, N., Higher level representations of quantum affine algebra $U_{q}(\mathfrak{g l}(2)), J$. Alg., 182 (1996), 448-468.

[LP] Lepowsky, J. and Primc, M., Structure of standard modules for the affine Lie algebra $A_{1}^{(1)}$, Contemp. Math., 45, AMS, Providence, 1985.

[LW1] Lepowsky, J. and Wilson R. L., Structure of standard modules I: Universal algebras and the Rogers-Ramnujan identities, Invent. Math., 77 (1984) 199-290.

[LW2] _ Structure of standard modules II : The case $A_{1}^{(1)}$, principal gradation, Invent. Math., 79 (1985) 417-442. 